\title{
Discussion on the Application of Complex Power Network to Software Engineering
}

\author{
Jiayan Song \\ Nanjing Institute of Railway Technology, Nanjing, Jiangsu, 210031, China
}

\begin{abstract}
Along with the further development of science and technology, computer hardware and the Internet are in a rapid development, and information technology has been widely used in all fields so that complex problems are simply solved. Because of the needs for the development, software starts to mutually integrate with complex power network, making the scale of software increase greatly. Such a growing trend of software promotes software development to go beyond a general understanding and control and thus a complex system is formed. It is necessary to strengthen the research of complex network theory, and this is a new way to help people study the complexity of software systems. In this paper, the development course of complex dynamic network is introduced simply and the use of complex power network in the software engineering is summarized. Hopefully, this paper can help the crossover study of complex power network and software engineering in the future.
\end{abstract}

Keywords-complex power network; software engineering; computer application; network model.

\section{INTRODUCTION}

With the constant progress of science and technology, the third technological revolution represented by electronic computer is changing the world in an unprecedented scale and influencing the world from all walks of life. With the rapid development of information technology and computer technology, software engineering was born at the right moment, and also software plays an increasingly great role in all fields and becomes an important tool to solve the problem of multiple changes. However, the limitations of the traditional software engineering development process have become the main problems of developers and researchers in recent years, as the information renewal is faster.

Software tends to mutually integrates with the Internet, bringing about a new development trend to the software system. On the one hand, software operation develops toward dynamics and multiple changes, but no longer based on a single machine. On the other hand, the purpose of software development is slowly oriented at network resource services. The traditional software engineering has been gradually unable to meet the requirements of the software complexity, so the application of complex power network to software engineering emerges. The introduction of complex dynamic network provides a new idea and way for the development of software engineering, which is of great help to the further development of software engineering.

\section{COMPLEX POWER NETWORK}

\section{A. The basic concept of complex power network}

In the world where people live, there exist a large number of complex systems, which have a common feature (they can be described by all sorts of network). The connection, which is composed of many nodes as well as connecting edges, is referred to as network. Nodes represent the different individuals in the real system, and a certain relationship between individuals can be represented with edges. Thus, the Internet, WWW, and medical neural networks in the real world and the interpersonal relationship network, work cooperation network, and science and technology citation network in sociology can be described by complex network. As a result, complex network exists everywhere in the life of people.

A complex dynamic network system contains a lot of subsystems. They have a complex relationship with each other within the whole system. A complex system can be abstracted into a complex power network, if these subsystems are many nodes and the mutual interaction between them is abstracted into the sides between nodes. The complexity of complex dynamical network is embodied in two aspects: (1) the number of the nodes of complex network is very huge, including large nodes; (2) there exist all kinds of connections between complex dynamic networks, which are generally very complex.

\section{B. The basic parameters of complex network as well as their meanings}

Understanding and exploring the basic parameters of complex network is very necessary to know well complex network. In the following, the basic parameters of complex network as well as their meanings are introduced.

1) The average shortest path length

In the network, the average shortest path length is obtained using the total distance between all nodes to divide by the total number of nodes. It is used for describing the degree of separation between all nodes. In complex power network, the message passing speed and the system response ability of a complex network can be measured based on the research of the average shortest path length. A deepening research and a major discovery found that the vast majority of large real networks have an average shortest path length much shorter than the expected, and this is the so-called "small world" phenomenon. The discovery of this phenomenon promotes the world to really stride forward the "global 
village".

\section{2) Aggregation coefficient}

Aggregation coefficient is used to show the tightness of the network. It can be used to describe the aggregation of the nodes in the network and accordingly measure the cohesion of different granularity software entities. A lot of practice has proved that the large-scale nodes in the real network tend to aggregate together and the aggregation coefficient is much larger than that of the random network. Large aggregation coefficient has become an important symbol of the modern software systems.

3) The degree of distribution

A particular node is first specified, around which the number of the adjacent nodes is called as degree. Usually, the network average degree refers to the mean value of the nodes in the network. Compared with other parameters, large-scale real network is in power-law distribution, but not normal distribution or Poisson distribution. The studies of the recent years found that like the large-scale real networks, power-law distribution can also be applied to the degree distribution of the nodes in the software network.

\section{THE APPLICATION OF COMPLEX NETWORK THEORY TO THE FIELD OF SOFTWARE ENGINEERING}

In recent years, the application of complex network theory to software engineering focuses on all kinds of complex software systems. Object-oriented software, open source software system, and WEB-based software system are the examples of its application. The actual research and statistical analysis are conducted according to the characteristics of complex network. Through measuring and testing large-scale software system, the currently applied software system is improved using the theory of complex network. At present, the theory of complex network is mainly applied to the following several aspects of software engineering.

\section{A. The modeling of software network}

The complex feature of network topology structure is a reflection of its essence, and it represents the network structure as well as advantage in the real cases. Many individual users in the network constitute a different real network under the condition of not being obstructed. These networks develop and evolve in a different environment, so that a relatively stable structure of complex networks is finally formed. Such a relatively stable network structure is a result of after a long investigation, which has been proven in the practical application. For this reason, software development, analysis, and improvement can be implemented according to such a mature and stable network topology, and the software system is explored according to the way of growth pattern to improve the algorithms and mathematical models.

\section{B. Software design}

The internal characteristics of software system are very complex, but also the main parts to decide the performances of software. In order to better develop software, getting a good understanding of the internal characteristics is a vital part, and a relatively perfect software system structure can be obtained by relying on the theory of complex network to guide its design. In the analysis and optimization of software structure, an improvement can be made according to the stability of the modeling, and also the spread of computer viruses can be analyzed. In the theory of complex network, the advantages of dynamics characteristics are very significant to guide and improve the structure design of the information transmission, and also the theory of community structure can be used to verify the structure modularity and optimize the data mining algorithm of network data, so that the speed and accuracy are improved.

\section{Software metrics}

Software metrics is a very important indicator, which can promote developers and designers to more objectively evaluate the quality of the software that has been developed. Thus, the shortcomings of the management in the development process can be eliminated, the resources are better allocated and utilized, and then a well-improved software development plan can be made. At the same time, the quality is very helpful for software developers to improve the working quality, summarize the problems in the work, and guide them to more rationally edit software. Thus, clear instructions are available for the developers in the further work. For the purpose of measuring the complexity of large software system, the structure and behavior of complex software system are quantificationally described and analyzed using the characteristics of complex network.

There are many methods for software metrics. On the basis of the element levels, degree distribution, aggregation coefficient, and the average shortest path length can be applied. However, analyzing complex network topology in the development of software system is a method common in software metrics at the present times.

\section{Software testing}

In late software testing, developers can analyze the relationship between the internal structural modules of software system and know clearly the important code segments and modules using the dynamic statistical applications of complex dynamic network, and then make more accurate test schemes according to the most important contents, so that the software development and testing can be effectively implemented. Currently, software testing now occupies a more and more important position in the development process. An important aspect to object-oriented test is to determine the test sequence. Effective test cases are necessarily chosen in order to achieve the requirements of test adequacy criteria.

\section{CONCLUSION}

In recent years, more and more software research and 
development personnel combine the theory of complex network with software engineering. Compared with the traditional software, network software coupling is getting looser, more autonomous, and scalable.

Software system is a very complex artificial system. In order to more quickly and efficiently meet the needs of users, the dynamic changes of complex network are necessary to constantly cooperatively combine together and the interactive changes serve for the application.

The key of applying complex power network to the development process of software system is to make a deep development, research, and measurement analysis of the network topology structure. Through the analysis of complex network model, the multiple changes and interactions in software system are made certain, and the problems and rules are better observed and discovered. On the basis of measurement, the quality of developed software system is effectively reflected in the appropriate software framework, and thus a powerful basis and reference is provided for the future software development.

\section{REFERENCES}

[1] Keqing HE, Yutao MA, Jing LIU, et al. Software Networks [M]. Beijing: Science Press, 2008.

[2] Shusen WANG, Qing GU, Dao CHEN. A Large Software System Measurement based on Complex Network [J]. Computer Science, 2009, 4 (2).

[3] Lei BAO, Fangwu YAO. The Design and Implementation of Assembly Source Debugger based on Remote Device [J]. Computer Technology and Development, 2009, 12 (11).

[4] Weihong LI. The Research and Implementation of Embedded Remote Debugging Tools [J]. Microcomputer Information, 2009, 25 (1 2).

[5] Deyi LI, Kun LIU, Yan SUN, et al. Emerging Computing: The Virtual Reality from Disorderly Applause to Orderly Applause [J]. Science in China, 2007 ((20).

[6] Jinhu LV, Hongchun WANG, Keqing HE. The Application of Complex Power Network to Software Engineering [J]. Journal of Computer Research and Development, 2008, 11. 